Noname manuscript No.

(will be inserted by the editor)

\title{
Information Aggregation and Computational Intelligence
}

\section{Limits to Price Discovery}

\author{
Shu-Heng Chen · Ragupathy \\ Venkatachalam
}

Received: date / Accepted: date

\begin{abstract}
This paper examines the possibility that the computational intelligence (CI) inspired tools can effectively aggregate the rich information generated from the Web 2.0 economy, and thereby enhance the quality of decision-making. Despite many advancements and commendable applications of CI in recent years, this issue has not been well addressed. We argue that this question is intimately related to the central issue of the socialist calculation debate since the time of Friedrich Hayek. In terms of information aggregation, we examine whether there is a better engineering than the market mechanism. More precisely, we focus on whether the CI-driven sentiment analysis can generate signals like prices and whether CI can process the unstructured text data better than the market. We argue that Web 2.0 economy may not be able to set us free from information overload problems that have long co-existed with the presence of markets. We attribute this to the tacitness and subjectivity of knowledge and the recursive (feedback) characteristic of the sentiments. In this sense, Hayek's fundamental assertion that the effectiveness of the market mechanism may not be so much conditioned on the information and communication technology still applies.
\end{abstract}

The first author and the second author are grateful for the research support in the form of Ministry of Science and Technology (MOST) grants, MOST 103-2410-H-004-009-MY3 and MOST 104-2811-H-004-003, respectively. We thank the two anonymous referees for their valuable suggestions that helped to improve the paper. All remaining errors are our own.

Shu-Heng Chen

AI-ECON Research Center,

Department of Economics,

National Chengchi University,

Taiwan E-mail: chen.shuheng@gmail.com

Ragupathy Venkatachalam

AI-ECON Research Center,

Department of Economics,

National Chengchi University,

Taiwan E-mail: rpathy@gmail.com 
Keywords Web 2.0, Computational Intelligence, Socialist Calculation Debate, Text Mining, Sentiment Analysis, Tacit Knowledge, Evolutionary Computation

JEL Codes: C63, B25, D8, C55

\section{Motivation and Background}

Fredrick Hayek broached an important theme in the middle of the last century that has had an envious and lasting privilege of being as relevant now as it was then. In attempting to characterize the fundamental economic problem faced by the society, Hayek (1945) called for a shift in focus. Instead of the optimal allocation of given resources, the relevant problem for him was about the ways and means for enhancing the efficient utilization of knowledge that is dispersed among the various members of the society. As the economies evolve and become increasingly complex in terms of the variety and scale of consumption, technological advancements and organization of production, the knowledge problem becomes increasingly relevant.

Along with the evolution of the economies, the technologies, especially information technology, have co-evolved. The introduction of the world wide web has had a profound impact on the economy and information organization. In 2004 the neologism, Web 2.0, was formally recognized and has been used to characterize the evolution of the Web up to that point and to foreshadow the extension to the foreseeable future. ${ }^{1}$

In 1970s, the founder of economic sociology, Mark Granovetter (Granovetter, 1973), indicated that, parts of market mechanisms, such as information flow and processing, were effectively carried out by social networks. Hence, the economy that existed before Web 1.0 can be viewed already as a combined operation of markets and networks. During Web 2.0, in terms of information pooling and dissemination, platforms with mobile internet and devices have constituted the market equivalent and social medium networks have become the social-network equivalent.

Nonetheless, the Web has constantly been evolving since then; different terms for the future of the Web have been suggested, such as Web 3.0, the Semantic Web and Intelligent Web. The extent to which the era of Web 3.0 becomes substantially different from Web 2.0 depends on the development of technologies such as sentiment analysis and natural language processing. These technologies are unique in the sense that they have facilitated the processing and understanding of unstructured information ${ }^{2}$, which was not possible earlier. More importantly, they focus on unearthing information such as sentiments, which are often implicit. Computational intelligence plays a critical role in this development. Tools from computational-intelligence are increasingly being applied to support text mining and sentiment analysis ${ }^{3}$ with the evolution of the Web 2.0 economy (see Section

\footnotetext{
1 The term Web 2.0 was popularized by Tim O'Reilly in 2004. O'Reilly and Battelle (O'Reilly and Battelle, 2009) provide a systematic guide to the origin and development of Web 2.0.

2 Unstructured information refers to the absence of a clearly defined data model, such as relational, hierarchical, network etc, according to which the information is organized.

3 The following can be regarded as an intuitive definition of sentiment analysis: 'Sentiment analysis or opinion mining is the computational study of peoples opinions, appraisals, attitudes, and emotions toward entities, individuals, issues, events, topics and their attributes. The task is technically challenging and practically very useful. For example, busi-
} 
5.1 for a brief review). This raises a fundamental question here : would there be a limit for these technologies? In this paper, we address this question by placing it within the context of the history of economic analysis.

We contend that examining the fundamental limits of these technologies and applications, if any, is essentially pertinent to the socialist calculation debate, a well-known episode in the history of economic analysis (Boettke, 2000). We then argue that, if such a limit does not exist, then Hayek's assertion regarding the theoretical impossibility of socialist calculation will be overthrown. For those who are skeptical about Hayek's impossibility thesis, in Section 4.1 we also elaborate on the notion of complex events or undescribable events, an idea borrowed from the modern incomplete contract theory, which may prove to be the fundamental limit.

A sketch of the paper is as follows. In Section 2, we begin with the mainstream view on the market mechanism for information aggregation, including information pooling and processing. We then move to the socialist calculation debate on the possibility of a supercomputer which can pool and process all information, and can do it better, including automating all key (economic) decisions. At this point, we review the classic work done by Friedrich Hayek, mainly Hayek (1945), regarding the impossibility of socialist calculation and the implication that markets remain the most effective mechanisms.

In Section 3, we address the following issue: in terms of information processing, in particular text mining and sentiment analysis, whether the current use of computational intelligence can be sufficient to represent the knowledge of Hayek's 'man on the spot'. We provide a general framework to be able to answer this question, which leads us to two refined facets related to the use of computational intelligence in sentiment analysis: 1) knowledge extraction or discovery (Section 4 ), and 2) prediction or predictability (Section 5). For the first facet, we argue that this difficulty can be better understood by relating it to Hayek's notion of the implicitness of knowledge and to the modern views on complex events or undescribable events in the incomplete contract theory. For the second facet, we address the theoretical and practical problems caused by the recursive structure (the feedback loop) of processed sentiments. In Section 6, we build on recent developments in the economics of attention to show that human attention has inherent limits. Despite the enormous progress in computational intelligence, information overload, information anxiety, and choice overload - phenomena well discussed in behavioral economics and library and information sciences - will still remain with the evolution of the Web 2.0 economy. Concluding remarks are given in Section 7.

\section{Knowledge, Market and Price}

In the history of economic analysis, Friedrich Hayek's work on the use of knowledge (Hayek, 1945) is a classic. This article is considered to be the one of 20 most important articles in American Economic Review in the 100 years since its inception (Arrow et al., 2011). It is a part of the famous socialist calculation debate

nesses always want to find public or consumer opinions about their products and services. Potential customers also want to know the opinions of existing users before they use a service or purchase a product.' - p. 415, Liu and Zhang (2012) 
(Boettke, 2000), which started in 1920 when Ludwig von Mises published his influential article on the impossibility of socialist calculation (Von Mises, 1935). This article (Hayek, 1945) has since been constantly examined in light of the later advancements in information and computation technology (Lavoie, 1985; Cockshott and Cottrell, 1997). We have little doubt that the significance of this article will continue to increase with the advent of Web 2.0 and the Semantic Web, and can shed light on the nature of sentiment analysis pursued by this volume. In this section, we shall first give a quick overview of this classic article as a foundation for the later analysis.

Based on Hayek (Hayek, 1945), a market is an institutional arrangement through which the knowledge of the particular circumstances of time and place can be "pooled" together. Such an arrangement implicitly indicate (rightly so) an interdependence among agents. However, it is not "pooled" 4 in the sense of being physically centralized but in the sense of a distributed network. In other words, market itself can be perceived as a network within which the knowledge of the particular circumstances of time and place can be preserved. Since such knowledge is almost never centralized spatially, the use of 'pooling' knowledge cannot be implemented by a 'central processor' or a central planner.

The reason for this is that the "data" from which the economic calculus starts are never for the whole society "given" to a single mind which could work out the implications, and can never be so given. (Ibid, p. 519; Italics added)

Prices are the emergent phenomena of the market or of the market when viewed as a network. They are an emergent phenomenon as Hayek clearly stated that these numerical or quantitative indices "cannot be derived from any property possessed by that particular thing, but which reflects, or in which is condensed, its significance in view of the whole means-end structure. (Ibid, p. 525)" Hence, they are derived from the knowledge of the particular circumstances of time and place, possessed by various 'men on the spot'. They are in fact results obtained after the "pooled" information has been processed. In this sense, they can be considered to be second-level knowledge or, based on the above quotation, the implications, which, we believe, constitute the essence of the sentiment analysis in many of its applications.

For Hayek, the market mechanism can facilitate the decision making of each man on the spot by economizing the amount of the knowledge that he or she is required to acquire, and this second-level knowledge is the answer to the following question that he posed:

Which of the events which happen beyond the horizon of his immediate knowledge are of relevance to his immediate decision, and how much of them need he know? (Ibid, p. 525; Italics added)

The most significant fact about this system is the economy of knowledge with which it operates, or how little the individual participants need to know in order to be able to take the right action. (Ibid, p. 526-527; Italics added)

It is then interesting to notice that Hayek applies the idea of a network to answer how much knowledge is required for each individual, even though a formal

\footnotetext{
4 Pooling and processing often assume that the information or knowledge is iid and not interdependent.
} 
notion of a network had not been introduced in his time. Hence, "it is always a question of the relative importance of the particular things with which he is concerned, and the causes which alter their relative importance are of no interest to him beyond the effect on those concrete things of his own environment. (Ibid, p. 525 ; Italics added.)" In the parlance of social networks, instead of six degrees of separation (Milgram, 1967), what we have here is only one degree of separation. This basically says that the network distance between the man on the spot and all the knowledge required for his specific decision involves only one degree of separation, even though the network distance between him and another man on the spot, who owns this knowledge, can be substantially long (see Figure 1 below). With these indices, each decision maker "will have to consider only these quantitative indices (or "values") in which all the relevant information is concentrated. (Ibid, p. 525; Italics added)" In a sense, these indices can be viewed as kinds of sufficient statistics. The required knowledge can be economized or the network distance can be shortened due to the existence of these emergent prices, indices or sufficient statistics (Figure 1).

In Hayek's analysis the entire price system can be considered as a second-level knowledge network, which in turn emerges from the bottom-level one (as shown in Figure 1). If one treats the first-level network as the network of agents, then the second-level one can be considered to be a network of symbols. ${ }^{5}$ As discussed above, the second-level knowledge network is a compressed version of the first-level knowledge network. With this successful compression, each agent in the first-level network has a way to harness the complexity of the entire world (the first-level network) by situating himself in the second-level network or connecting himself to it, as shown in Figure 1.

Figure 1 can considered to be the essence of Hayek (1945). The famous Hayek hypothesis, well formulated by experimental economists (Smith, 1982), is demonstrated via this figure: the symbols at the second level "can act to coordinate the separate actions of different people in the same way as subjective values help the individual to coordinate the parts of his plan. (Ibid, p. 526)"

What has not been addressed in Hayek (1945) is the whole emergent process of those non-price symbols, and the associations of these non-price symbols with each 'man on the spot'. It is, however, quite plausible to endow Figure 1 with an adaptative and evolutionary process, such as evolutionary computation, which constantly reviews and revises the existing symbols and hence generates new symbols for the 'man on the spot' to form and "fit his decisions into the whole pattern of changes of the larger economic system (Ibid, p. 525; Italics added)" .6 In fact, Nicolaas Vriend (Vriend, 2002) proposed an agent-based model of customer reviews to simulate this process.

However, those who praise Hayek's analysis of the use of knowledge often ignore the significance of the network topology of the first level (network of men). In fact,

5 Hayek did use the term 'symbol' in his discussion on the marvels of the price system. "In abbreviated form, by a kind of symbol, only the most essential information is passed on, and passed on only to those concerned. (Ibid, p. 527; Italics added.)" A broader interpretation of prices as symbols makes it easier to see the relevance of Hayek's analysis to the modern Web 2.0 economy, in which the use of the sentiment analysis to the big data available in the social media network, such as FinanceTwitter, normally leads to many linguistic-type indices, rather than numerical prices.

6 A detailed discussion of this feasibility is beyond the size of a chapter. The interested reader is referred to Chen (2015); specifically, those models involve the use of sunspot variables. 


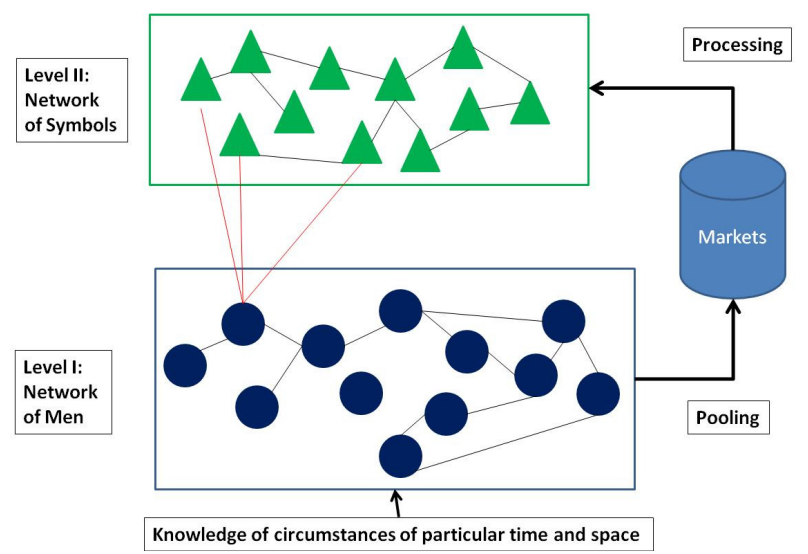

Fig. 1 The Two-Level Knowledge Network: From "Man on the Spot" to Symbols In the figure above, the second-level knowledge network emerges from the bottom level. These include the emergence of the symbols (prices and indices) and their connection. At the first level, the "man on the spot" may be a long distance away from the other "man on the spot" who owns knowledge of specific circumstances of a particular time and place. However, given the availability of the indices at the second level, the man at the first level can get direct access to those symbols at the second level in one degree of separation. Hence, the appearance of the second-level network bridges the gap between men in the communication and use of knowledge.

possible reservations on what is called the Hayek hypothesis are exactly rooted here. As Hayek argued,

The whole acts as one market, not because any of its members survey the whole field, but because their limited individual fields of vision sufficiently overlap so that through many intermediaries the relevant information is communicated to all. (Hayek (1945), p. 526; Italics added)

The pre-condition for the validity of the Hayek hypothesis, therefore, depends on whether the first-level network sufficiently overlaps. Using the current state of social network analysis, one can examine this condition by checking whether there are enough redundant links connecting nodes or the density of networks or the number of isolated nodes or components, etc. The key is that the extent of development of the second-level network depends on the topological structure of the first level.

Armed with advancements in information technology, technologies like sentiment analysis can be seen as generating a list of price equivalents, called indexes, indicators, or sensors, which basically have the same informative function as that of prices. However, the difference lies in the origins, i.e., by whom these "prices" are generated. To Hayek it is those 'men on the spot' who collectively generate these prices. In the economy of web 2.0, these "prices" can be generated in a similar manner by the wisdom of the crowd, such as crowdsourcing or Amazon Mechanical Turks; however, they can also be generated by machines or computational-intelligence algorithms. The question then concerns the difference between the "prices" generated by men and the "prices" generated by machines. Are they equivalent? 


\section{Dispersed Knowledge, Computational Intelligence and Omniscience}

There has been an unprecedented advance in the way we communicate with each other and how we search, gather and store information with the evolution of the internet to web 2.0. With the rapid spread of hand-held devices and social media, more storable data is being generated now than ever. Concurrent developments in computational intelligence, increased computing power and storage capacities have placed enormous amounts of data and processing power at our disposal. Unlike the era that preceded this, a lot of data which are user-generated are often unstructured, often in the form of text that is not immediately amenable to processing and distilling. Text mining, or text analytics as it is referred to sometimes, is one of the developments that addresses this gap by enabling the businesses and various other types of users to extract potentially useful information from vast amounts of data.

\subsection{Text mining and sentiment analysis}

In this section, we aim to provide an intuitive characterization of text mining and sentiment analysis. Text mining aims at discovering patterns and extracting useful, 'high quality' information from unstructured, often user-generated data. It differs from information retrieval in the traditional sense that uses keyword search. According to Aggarwal and Zhai (2012a), "[T]ext mining can be regarded as going beyond information access to further help users analyze and digest information and facilitate decision making (Ibid, p. 2)". While information retrieval involves providing the user with documents that match the keywords in his query as it happens with Google search, information extraction goes a step further by extracting and compressing semantic information from text data. This is accomplished by a combination of tools and insights from natural language processing and computational linguistics, augmented by computational intelligence. They involve both rule-based and probability-based approaches ${ }^{7}$ and a detailed survey of different tools that are employed in text mining can be found in Nenkova and McKeown (2012), Aggarwal and Zhai (2012b), and Sun, Deng and Han (2012). The potential applications are diverse, ranging from extracting information regarding new discoveries in biomedical research, gathering information and outlooks that may be useful for finance professionals, to crucial information gathering for intelligence and security services.

Sentiment analysis, on the other hand, has been an active topic both in academic circles and also in commercial arenas (Liu and Zhang, 2012). It has applications in the fields of economics, marketing, political science and even the prediction of election results. Shifts in sentiments held by the agents are known to have significant impact on the aggregate economic outcomes and fluctuations in economic activities (Angeletos and La'O, 2013; Mian, Sufi and Khoshkhou, 2015). Understanding these shifts in market beliefs or the over all sentiments is key to

\footnotetext{
7 The use of statistical tools to understand patterns in textual data has a long history in linguistics. In 1930s, the American linguist George Kingsley Zipf studied the distribution of words in natural language (Zipf, 1936, 1949) and observed that there was a proportional relationship between the frequency of word occurrence and its rank in the frequency list. This relationship is now popularly known as the Zipf's law.
} 
making predictions about the economic outcomes. Financial markets present an excellent example about the relevance of understanding sentiments.

These developments do have many practical applications and positive uses for society. While acknowledging their usefulness, we attempt to address a slightly different question. Given the rapid advances, we are interested in the extent to which these methods can be of help in solving the fundamental problems of knowledge aggregation. It is natural to expect that these technological developments may lead us to surpass inherent limitations regarding economic prediction, public opinion aggregation and, potentially, even for extensive applications in the domain of public policy.

\subsection{Omniscience and Computational Intelligence}

In the light of the discussion in Section 2, we can interpret these developments as generating 'symbols' by pooling knowledge and extracting 'high quality' information. Earlier, we presented a distinction between pooling achieved by agents in a network and that accomplished by a centralized authority (or intelligent algorithms). We can question whether these developments in computational intelligence can overcome the problems that might plague a central planner. Can these advancements rooted in advances in computational intelligence help achieve a society that uses information and knowledge effectively? This is equivalent to asking whether computational intelligence, manifested through tools like sentiment anlaysis and text mining, has the potential to constitute an omniscient planner.

We distinguish three aspects that may be relevant for assessing the potential for achieving such an omniscient planner:

- pooling dispersed knowledge,

- extraction or summarization of high-quality information from pooled knowledge, and

- prediction based on the summary.

Limitations concerning each of the above aspects, even while acknowledging their usefulness, need to be examined.

\section{Knowledge Pooling and Aggregation}

Data mining in general, and text mining in particular, is about information extraction as opposed to retrieval and this feature poses interesting questions to a social scientist. For instance, sentiment analysis involves extracting information regarding the general opinion concerning a product, person, or more generally any specific item of interest. These opinions can be synthesized to express whether they are positive or negative overall, in other words, express how polarized they are. ${ }^{8}$ While this may be useful for market surveys and some aspects of research, indiscriminate applications to more complex, sensitive domains such as public policy and finance should be seen with caution.

\footnotetext{
8 This can be taken to further granular or advanced levels such as 5-point scale for classifying the opinions based on more precise moods (supportive, confused, excited, sad and so on).
} 
This involves understanding whether or not the relevant knowledge - be it objective information or subjective opinions - that is scattered among various agents can be reliably pooled, extracted and summarized by recent advances in computational intelligence. It also involves examining the possibility that these technologies constitute the magic recipe for information aggregation to be reliably employed in, say, policy making. It is fair to say that such questions cannot be answered in binary terms and it is often a matter of degree. Even if we acknowledge this admonition, we argue that the domains and the extent of this possibility are limited for several reasons. In the remainder of this paper, we focus on the domains of economics, finance and policy making.

\subsection{Tacit Knowledge}

Computational Intelligence inspired methods like sentiment and text mining involve pooling, aggregation and summarization of information either from social media or a corpus of textual data. Their primary goal is to discover patterns, in other words, 'new knowledge'. We need to consider the nature of pooled disaggregated data from which useful information needs to be extracted. Often, there are aspects of knowledge that are only in the minds of individuals, which are difficult or even impossible to articulate. The distinction between knowledge that can be codified and that which cannot be was made popular by Michael Polanyi (18911976) (Polanyi, 1966). The latter is also known as tacit knowledge. Following his argument that 'we can know more than we can tell', there is a long-established awareness both in philosophy of science and in organizational studies that there are various aspects of personal and individual knowledge that cannot be codified in formal or linguistic terms. However, this kind of knowledge may be extremely important.

A related notion that captures this aspect of knowledge is the idea of undescribable events or complex events that is invoked in one strand of the literature on incomplete contracts (Hart and Moore, 1999; Tirole, 1999; Aghion and Holden, 2011). When parties write contracts with each other, not all contingencies or intentions can be described in terms of a finite description (Al-Najjar, Anderlini and Felli, 2006). By relating the notion of feasible description to finite description, which is clearly relevant in the case of knowledge description, the notion of complex events signifies the limit to description. Even if such articulation were possible, it can suffer from imprecision when it is executed by means of natural language. Therefore, text mining and related approaches, in the light of tacit knowledge, have a natural limitation in what they can achieve in precisely extracting and summarizing dispersed knowledge and collective opinion.

Along with the plausibility of pooling all existing, relevant knowledge, the process of aggregation or extraction of 'high quality' information is also important. Mining methods strive to unearth 'new' insights about social processes and economic behaviour that could potentially inform decision makers - be they businesses or policy-makers. In practice, any process of statistical aggregation typically involves some loss of valuable information (Section 2). This consideration is amplified in social environments involving sensitive judgements, especially when coupled 
with the difficulties of semantic ambiguities posed by natural language. A further complication may arise when texts deal with highly subjective expressions. ${ }^{9}$

\subsection{Subjectivity}

In addition to the possible subjectivity of the extracted knowledge, the role of the human who interprets this knowledge cannot be ignored as well. A crucial function of text mining methods is that they aid in information compression and pattern discovery. This is highly relevant in environments where individuals are boundedly rational, with limited cognitive capacity and attention, have to deal with large amounts of data and especially when the data are unstructured and textual. Knowledge discovery in this context often involves using syntactic or statistical approaches (such as the bag-of-words or n-grams approach) by which a corpus of text is often transformed into numeric vectors ( $\mathrm{Hu}$ and Liu, 2012).

Once this is achieved, computational intelligence can be used in unearthing or mining knowledge that resides in these texts. Since these methods are algorithmic and statistical, there is often a mistaken belief that the extracted knowledge is objective or 'true'. ${ }^{10}$

However, it is important to note that linguistic expressions, especially those expressed in social media, are highly contextual and their inherent subjectivity may never be eliminated completely. In addition to this, we need to also emphasize the subjectivity, cognitive limitations and biases of the recipient (financial analyst or policy maker) who interprets this compressed information.

To claim that the dynamics of human interaction and the complexity of the social world can be reduced to a self-explanatory set of nodes and edges defies important insights from fields as diverse as machine learning, sociology, and economics. Data sets are not, and can never be, neutral and theory-free repositories of information waiting to give up their secrets. They require the active interpretation of researchers, all of whom have their own ways of seeing. (Crawford, Gray and Miltner (2014), p. 1668)

Given this 'double subjectivity', hopes that mining methods, or big-data methods in general, can successfully solve all or most problems of public policy have to be measured. In the case of sentiment analysis, we measure the polarity of opinions that people hold over a single, specific issue or an attribute. If this were to be generalized to handle opinions over many attributes, a reliable framework for preference aggregation may be necessary. For text mining or opinion analysis to be an effective input in policy making, such reliable aggregation techniques have

\footnotetext{
9 However, we do not diminish the achievements made in processing opinions or subjective text over the years; see, for example, Liu and Zhang (2012), Sections 3-6.

10 For instance, Liu and Zhang (2012) notes that

Moreover, it is also known that human analysis of textual information is subject to considerable psychological biases, e.g., people often pay greater attention to opinions that are consistent with their own preferences. People also have difficulty, owing to their mental and physical limitations, producing consistent results when the amount of information to be processed is large. Automated opinion mining and summarization systems are thus needed, as subjective biases and mental limitations can be overcome with an objective sentiment analysis system. (Ibid, p.415-416; Emphasis added.)
} 
to be uncontroversial. However, opinion aggregation, or more generally judgement aggregation, is far from being a trivial matter, as can be seen from Dietrich (2006), List (2012), and Bozbay, Dietrich and Peter (2014).

\section{Prediction and Sentiment: The Role of Feedback Effects}

Until now, we have been focusing on the feasibility of information/knowledge aggregation and extraction in mostly static environments from the viewpoint of a social science research. However, further considerations may arise when we move on to dynamic environments. In this section, we extend our focus on dynamic environments. There is now an increasing consensus that the economic and social systems are best viewed as complex, dynamic environments in which heterogeneous agents interact among each other. In this characterization of a society as a complex adaptive system, the role feedback effects, nature and the modes of information dissemination are non-trivial. This is particularly relevant in the fields of economics, finance, political science and management science, where the presence feedback effects may lead to drastically different outcomes.

The explosion in the scale of user-generated data in social media that is now available for analysis has been welcome news for those interested in data-driven approaches in various fields. CI-aided tools have made commendable advances both in gathering and summarizing unstructured data that was not possible a few decades ago. Applications are diverse, ranging from market-structure surveillance (Netzer et al., 2012), predicting public health (Dredze, 2012), labor market flows (Antenucci et al., 2014), near-term economic activity (Choi and Varian, 2012), stock markets (Bollen, Mao and Zeng, 2011) and election outcomes (Tumasjan et al., 2010).

These advancements raise questions regarding the possibility of exact predictions of elections, political outcomes, financial markets ${ }^{11}$ and so on, with the aid of computational intelligence. So far, the claims about the effectiveness of these tools have been mixed. For instance, in the case of electoral predictions, it has been challenged in the literature on the grounds that twitter data-based methods are not an efficient, consistent predictor of electoral outcomes, at least as of now (Metaxas, Mustafaraj and Gayo-Avello, 2011; Gayo-Avello, 2012). On the other hand, applications to other areas such as financial markets have been claimed to be relatively successful.

\subsection{Financial Markets and Sentiment Analysis}

Financial market prediction, in particular, has become an increasingly popular domain related to the application of computational intelligence tools to textual data over the past few years. In the era of digitization and text mining, information concerning sentiments is extracted from different news and this representation has been used in automated trading algorithms (Mo, Liu, and Yang, 2015). The nature

\footnotetext{
11 It should be noted that the possibility of 'emergence' is another aspect of these systems that make prediction hard, even in theory. For instance, if the changes or shifts in beliefs held by agents in a market or a society are themselves 'emergent', then predicting them based on the information concerning micro-states (agents and their tweets) can be a difficult task.
} 
and impact of these quantitative measures of sentiments, especially in the light of developments in big data, are yet to be fully understood. It may be useful to describe the approaches that are currently employed in a bit more detail in order to get a better, overall picture.

Financial news articles are often utilized by investors to gauge the 'mood' of the market and ultimately to assist in making investment decisions. Behavioral Finance, in particular, acknowledges the role of sentiments in the possible deviation of prices from their so called 'fundamental' value. Therefore, gaining insights into investor sentiment and quantifying them can possibly help investors predict stock price movements, market performance and volatility (Tetlock, 2007; Tetlock, Saar-Tsechansky and Macskassy, 2008). Given the increasing volume of news that is generated, for users and news agencies alike, there is an increasing need for comprehending and extracting a useful measure of market sentiments. This is where the role of computational intelligence tools such as learning algorithms and classifier systems come into play: to extract and learn the necessary signal from the noise.

Apart from using survey-based and market variable-based measures of sentiment, studies also use quantitative proxies that are extracted from social media and internet message boards. Computational intelligence tools are used to extract information from millions of downloaded messages from the websites such as Yahoo! finance (Kim and Kim, 2014). For example, a naive Bayes classification algorithm is frequently used to obtain a 'bag of words' representation, where text messages are split into words (features) and the text messages are associated with a unique label (buy or sell, for example). The conditional probability of a given label, assuming that the features occur independently of each other, is then obtained. These classification algorithms are typically trained using a smaller dataset before they are applied to a larger database. Indices of investor sentiment can, in turn, be constructed based on this classification. In essence, they relate the statistical pattern of word occurrence to the dynamics of the stock price and explore this relation.

Other studies, such as Li et al. (2014), use an approach slightly different from the 'bag-of-words' approach by constructing a sentiment space using sentiment dictionaries such as the Harvard psychological dictionary and Loughran-McDonald financial sentiment dictionary. In Li et al. (2014), texts and news articles are then projected into the sentiment space. They are then classified using support vector machines as the learning algorithm. Other studies use support vector regressions, K Nearest Neighbors, etc. ${ }^{12}$

It may be useful to distinguish some aspects of the prediction possibilities at the outset. Predictions can be approximate, accurate or probabilistic. In using twitter or other social media trends, one essentially searches for an equivalent of a sufficient statistic that would be of use in an approximate or probabilistic prediction. ${ }^{13}$ A more refined statistic that captures public moods, say, Facebook likes or the intensity or volume of specific twitter hashtags, may provide useful information in narrowing down the approximation. Accurate or exact predictions, as the name suggests, occur when the predictions and outcomes exactly coincide.

\footnotetext{
12 See p. 828, Tables 1 and 2 in Li et al. (2014) for a useful summary.

13 See Murthy (2015), who argues that twitter feeds play more of a reactive rather than predictive role in elections.
} 
We now examine the case of electoral predictions, which caution us about placing such excessive hopes on both approximate and accurate predictions. A similar argument can be extended to financial markets as well. We later analyze a hypothetical case relevant to the popular restaurant feedback scheme, highlighting the role of feedback effects in both situations.

\subsection{Predicting elections: How accurate can we get?}

The possibility of accurate predictions of social and political outcomes has been a topic of interest for a long time. Scholars have explored both the theoretical possibility and the empirical feasibility of predicting, among other things, election outcomes. One reason for the potential difficulty in prediction is the presence of interdependencies among agents. In the light of our earlier discussion, we ask whether computational intelligence methods that classify sentiments can help us predict polls perfectly.

\subsubsection{Predictability 'in principle'}

Almost 60 years ago, Herbert Simon posed and addressed an important question concerning the prediction of electoral results (Simon, 1954). Often we come across opinion polls that survey voters and based on the results from these sampled voters, polling agencies attempt to predict electoral outcomes. The interesting issue here is the possible effects of this act of publication concerning poll predictions on electoral outcomes. Simon highlighted two possible effects that are of interest, viz., the bandwagon effect and the underdog effect.

The bandwagon effect points to a behavior where the voters tend to choose a candidate who they expect to win. The underdog effect, on the other hand, is at play when the voters choose to support a candidate by voting for him because they expect him or her to lose. In both these cases, the voter's decision depends on the 'expected' outcome. Hence, it could be argued that the publication of the opinion poll results could lead to a change in voter preferences, which in turn changes the election outcome from that of the prediction. Apart from the possibility of manipulation, this raises questions about the very possibility of predicting outcomes in the social sciences, where information feedback can cause a change in behavior and hence destabilize the original prediction.

Herbert Simon demonstrated that even in the presence of these 'publication' effects accurate predictions are indeed possible, in principle. That is, Simon showed that the predicted and the actual vote can, in principle, be made to coincide by a poll prediction agency. He used a fixed point argument, more precisely, Brouwer's fixed point theorem for his argument. However, it should be noted that Brouwer's fixed point theorem relies on a non-constructive proof and hence does not offer a procedure or a mechanism by which the poll agency can consistently achieve such an accurate prediction. In fact, the validity of Simon's argument that perfect predictions are possible in principle was a subject of an interesting debate between him and the mathematician Karl Egil Aubert (Aubert, 1982a; Simon, 1982a; Aubert, 1982b; Simon, 1982b; Aubert, 1987).

While the concerns about the relevant nature or the data domain that Aubert raised are partly addressed by replacing Brouwer's fixed point theorem by a re- 
cursion theoretic fixed point theorem by Kleene, as suggested in Velupillai (2010), pp. 164-173, we still lack an explicit construction, procedure or an algorithm for such an accurate prediction based on opinion aggregation. ${ }^{14}$ This is because the computable function in the recursion theoretic fixed point theorem is only implicit. Although these concerns are about accurate prediction in principle, it is evident that this is neither straightforward nor can be taken for granted. Questions about the feasibility of a universally valid procedure for accurate predictions across domains still elude us.

\subsubsection{Predictability 'in Practice'}

How about in practice? For a robust argument about the presence or absence of inherent limits to electoral prediction, demonstrating a theoretical possibility alone may not be sufficient and we may need to look at the empirical evidence. For instance, we need to look at the evidence concerning the presence and the extent of the bandwagon and underdog effects to see the extent to which this issue may be relevant in practice. Otherwise, one can be justified in brushing this aside as 'much ado about nothing'. Although it is a challenging issue to empirically pin down the existence and magnitude of the bandwagon effect, recent empirical studies do confirm the presence of this effect in the past (Hodgson and Maloney, 2013) and also in more recent elections (Kiss and Simonovits, 2014). The potential of these poll predictions to alter election outcomes, and possibly the scope for manipulation, has led countries like France to ban the publication of opinion poll results in the days preceding the election. In other countries, like India, where the elections are staggered, the publication of exit poll results is banned when the election is under way.

Among the possible trade-offs while using text mining techniques, one involving the availability (cost of information acquisition) and the quality of the information is important. In particular, researchers point to the measure of 'veracity' of the information so as to make reliable inferences (Lukoianova and Rubin, 2014). This issue is not trivial when dealing with textual data from social media, which is often subjective and uncertain (in terms of content and the form of expression).

The veracity of the data is further complicated by agents who are strategic. For example, when agents are aware of the feedback of the information they provide back into the system, they can manipulate or trick the system by responding strategically. As long as there is heterogeneity in terms of the agent population, learning methods and awareness, there would be a potential scope for strategic manipulation, be it in terms of swinging opinions during elections, attracting customers for restaurants and so on. In such cases, the population may end up being worse-off on the whole, despite or even because of these technologies.

So far we have analyzed whether perfect prediction can be achieved with the aid of computational intelligence-inspired tools. These intelligent learning and classification algorithms are seen as the 'omniscient planner' who can pool and extract information seamlessly. This as we pointed out earlier is intimately related to the socialist calculation debate. We now turn to the case where users themselves generate these indices and learning algorithms merely in order to summarize this information for other users.

\footnotetext{
14 See Otnes (2011) for a detailed discussion on this issue.
} 
An important aspect of information aggregation that is relevant for this context was pointed out by Cass Sunstein (Sunstein, 2008) in response to the claim by Richard Posner that blogs had the 'potential to reveal dispersed bits of information' (Posner, 2004). In addition to the fact that the opinions shared in social media, blogs, and twitter are subjective and the tacit dimension of knowledge that cannot be written down, there is no equivalent of a coordination device in the case of text mining, as in Hayek's example. This lack of an 'effective' coordinating device, such as the price system, implies that the there is no guarantee that the incentives and actions of the players are appropriately aligned in a dynamic context. Hence, text or sentiment mining as a mode of information and knowledge aggregation are crucially their limited efficacy, even if we choose to employ a Hayekian framework of decentralized knowledge to understand this phenomenon.

In the light of these theoretical and empirical considerations, we are now in a position to answer the question we posed at the beginning of this section: can text analytics, sentiment analysis, and big data methods in general circumvent these impediments to accurate prediction of economic, financial and political outcomes? Our view is that computational intelligence can supplement, improve or even replace traditional sampling methods of opinion analysis and predictions with the ever increasing user-generated data. However, while they can possibly help increase the level of approximation in prediction, the issues concerning the diversity of feedback effects (partly due to heterogeneity in the population) means that the possibility of a perfect forecast will still remain.

\subsection{Publication of Reviews: Always Better?}

Information extraction by CI-inspired tools can be of use to agents provided they equip them with the ability to make better decisions. These tools aim to extract information from a corpus of data in the form of relevant summary statistics or indices. These indices are geared towards potentially aid users to make intelligent or smarter decisions. There are at least two reasons why this function may be considered useful and the role of computational intelligence tools in facilitating this is certainly commendable.

First, given limited cognitive capacity and time, it would be a monumental task for an individual to sift through all the data and obtain the relevant information, such as, perception regarding a product or a policy. Hence, the availability of smarter, intelligent tools that extract this information, summarize and present it to the decision maker in a readily usable form is a welcome step forward. Second, to do this at a relatively low cost and at a quicker pace means a higher level of adaptability to the dynamic environment. It may even indicate a competitive edge for businesses when they are able to make such data-driven decisions. For example, consider the analysis of how customers feel about a particular product like a smartphone or a speaker. Sentiment analysis can help provide companies with valuable insights regarding what customers feel in general about the product and whether there is any particular attribute or feature that the users feel negatively about or disappointed with.

Having described centralized information extraction out of a decentralized pool of texts, we now turn to user-generated metrics (or symbols). Restaurant reviews and movie reviews that are widely available can serve as good examples. These 
reviews are used by other actors in the system to make decisions. Similarly, one can think of restaurant reviews in a city like New York or London, where there is an ample supply of options and the decision maker can simply feel overwhelmed. In addition, there is the very important decision about how to choose a movie on a Friday night! But, can such metrics or sufficient statistics always be relied on to make decisions? Are they (and the underlying mechanisms of aggregation) invariant over time? Can they have adverse effects, given the feedback possibilities? These questions concern the impact of computational intelligence tools when they become an active input in to decision making. A relevant case is that of financial markets, where feedback from news and investor sentiment has important effects. For instance, employing investor sentiment indices have the possibility of amplifying 'herding' behaviour. Nicolaas Vriend (Vriend, 2002) provides some important insights concerning this issue.

Using an agent-based model, Nicolaas Vriend studies a decision problem that involves a choice between finite items (two in his case) of different quality, which have stochastic payoffs. Individual decision making is modeled using John Holland's classifier systems that consist of a set of if-then rules (Holland, Holyoak and Nisbett, 1986). These rules range from following the majority to average rules, random rules and so on. This caricature model can be a tool used to understand the choice of movies, restaurants, and cafés based on user-driven online reviews. We can ask whether the rules of choice that follow user-generated reviews always perform well. Note that there are many ways in which these reviews can be sampled or aggregated. What is their overall impact in guiding users to make efficient choices? This is precisely the question that Vriend sets out to answer using his model. He finds that, over time, there is a tendency for these agents to self-organize themselves towards some specific rules. This is due to the fact that there are lock-in effects and the evolution is path-dependent. This indicates that there are non-trivial feedback effects that may or may not be welfare enhancing for the society as a whole. Hence, dependence on quantitative measures derived from user-generated reviews' sentiment analysis may not necessarily help users to always make efficient choices in inter-temporal situations.

Even if they do work in certain situations, there is no guarantee that they will work consistently. This is partly because of endogenous reactions due to feedback and partly due to the fact that these methods are less concerned about unraveling causal factors, instead focus disproportionately on prediction. This concern was exemplified by the episode involving the failure of Google Flu Trends (Butler, 2013). Thus, we see that even with user-generated indices, the possibility of feedback may create interesting, complex dynamics.

\section{Information Overload}

Based on the above analysis in Sections 4 and 5, we consider two contrasting cases, namely, the left and the right panels of Figure 2. At both ends of the two panels, we see a deep blue cylinder, which signifies the richness of the data in two societies. The left and higher cylinder of both panels corresponds to a big-data society, say, the Web 2.0 society, whereas the right and the lower cylinder of both panels corresponds to a small-data society, say, the Web 1.0-or-lower society. The sharp difference in the heights of the two blue cylinders indicates the dramatic leap 

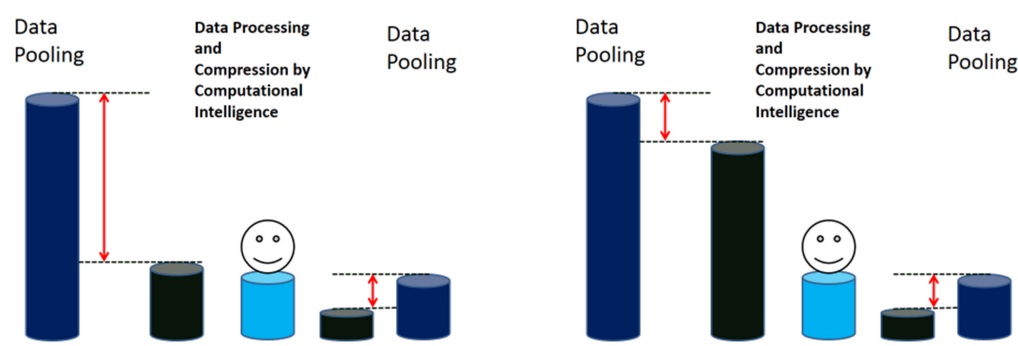

Fig. 2 Generated Data and Extracted Information: Web 1.0 and Web 2.0

In both panels, the Web 1.0 society is taken as a benchmark and its generated data (dark blue) and extracted information (dark green) are shown at the right end. The two panels differ only in their left ends. The left end of the left panel corresponds to the user-generated data with lower complexity, whereas the left end of the right panel corresponds to the user-generated data with higher complexity. The light-blue cylinder below the smiling face emoticon in the middle denotes the cognitive capacity (the attention capacity) of the agent who is furnished with the extracted information.

from one society (Web 1.0) to another (Web 2.0) in terms of the data generated. Not all the pooled data are useful, and they can be extracted or compressed using, for example, computational intelligence tools.

If the data generated by the Web 2.0 society has a simple structure (the left panel), and hence a low algorithmic complexity (Kolmogorov complexity), then the pooled data can be substantially compressed and the height of the cylinder after data compression, as shown in dark green, becomes much smaller. On the other hand, if the data generated by the Web 2.0 society has a complex structure (the right panel), and consequently greater algorithmic complexity, then the height of the cylinder after data compression remains high, as it is shown in dark green. By comparing the above two cases with the benchmark, i.e., the Web 1.0 society (the right end of both panels), both cases (the left and right panels) have additional information after extraction. However, the latter (the right panel) has exceedingly more.

Now, does having more information necessarily constitute a gain? Based on the literature on the information overload hypothesis (Schroder, Driver, and Streufert, 1967; Jacoby, Speller and Kohn, 1974; Jacoby, Speller and Berning, 1974), we know that the answer depends on the attention limit or cognitive capacity of agents (Simon, 1971). We also illustrate this difference using Figure 2. A typical agent with his cognitive capacity is shown in the middle. The height of the light-blue cylinder captures the cognitive capacity or information processing ability of the agent. Whether the additional information can bring a gain to the agent depends on whether he is able to process it. It is a gain only if he can process it; otherwise, it is an overload. By this criterion, we can see that the additional information in the left panel is a gain, but that in the right panel has crossed beyond the processing limit of the agent, which may signify a loss based on the information overload hypothesis, as shown in Figure 3.

Figure 3 depicts the information overload hypothesis. In the initial stage, information load may help decision makers in terms of their decision quality (accuracy); however, it can do so only up to some point (peak), say, $x_{\max }$. There is an inverted U-reversal indicating that any further information load may reduce the decision quality, due to a cognitive deficit to process this excessive amount of information. 


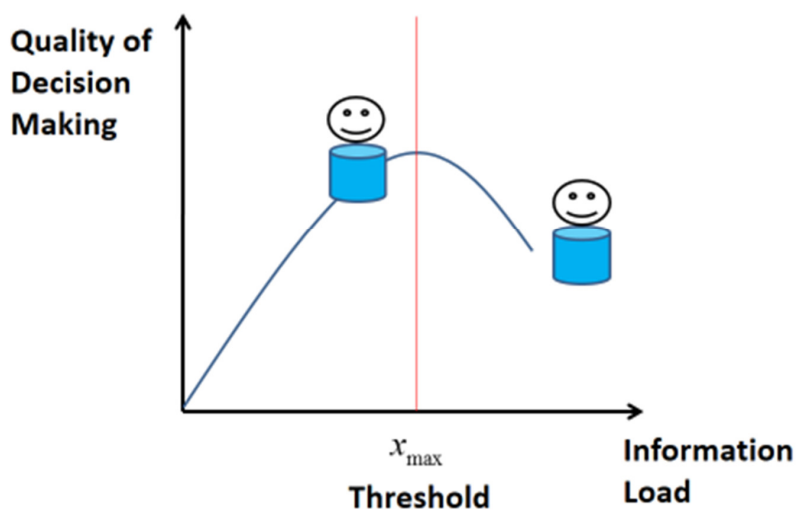

Fig. 3 Information Overload

The stage after the inverted U-turn is then perceived as a stage of information overload.

Hence, even though the big data characterizing the Web 2.0 economy can be processed by computational intelligence, given its lack of structure and the prevalence of ambiguity and subjectivity, there is no guarantee that this information can be condensed in such a way that the extracted information can be processed effectively by human beings. In fact, this is relevant as more and more consumers use Web 2.0 tools, such as online discussion forums, consumer review sites, weblogs and social network sites, to communicate their opinions and exchange product information. This new form of word-of-mouth (WOM), known as the electronic WOM, has now become another source of information overload (Park and Lee, 2009). Therefore, as shown in Figure 3, although the extracted information available for decision makers is less in Web 1.0 (the left smiley emoticon), its decision quality can be superior to that in Web 2.0 (the right smiley emoticon), where agents are burdened with information overload.

\section{Concluding Remarks}

Despite the limitations that are indicated in this paper, these developments in CI certainly constitute an interesting step forward. While the idea of computing with words had already proposed and promoted by Lofti Zadeh at the turn of the 21st century, the recent advances in big data manifested in various forms of unstructured texts in the social sciences that have made the alphabet, sign or symbol become the quarks in social sciences. About a century after Ferdinand de Saussure (1857-1913) and Charles Peirce (1839-1914) founded semiology or semiotics, we can see a resurgent interest in the study of symbols as a part of social life. The Web 2.0 economy has drawn our attention to the emergence of new symbols (terms, keywords), sets of symbols, networks of symbols, and their diffusion and dynamics.

The meaning and sentiment of a symbol or set (network) of symbols is derived from the contexts within which they are embedded. Over the last few years, computational intelligence has made noticeable progress in "discovering" this correspondence. In this paper, we made an attempt to understand the possibilities 
and limitations of using these technologies to enhance decision making that are relevant for various economic and social situations. As shown in Figure 1, the system of symbols is constantly co-evolving with the society of agents. Hence, in addition to knowing the generated sentiments with a set (network) of symbols at a given point in time, one cannot ignore the fact that these symbols either individually or collectively are constantly competing with others with respect to a given sentiment, idea, or affection. On the other hand, various sentiments, ideas, and affections are also competing for their proper symbolic representations. This entire evolutionary process can be further complicated as it is part of the evolution of the culture, social norms, and demographical structure. Historical incidents can also perturb its trajectory. In sum, the mapping from symbols to meanings is itself rapidly evolving.

Instead of applying computational intelligence to uncover the mapping by naively assuming a stable scaffolding, one should have a clear and broader picture of the dynamics underpinning the system of symbols. Evolutionary computation, one major tool-kit of computational intelligence, has the potential to study this metaphysical issue (Akhtar, Koshul and Awais, 2013). Among a number of possibilities in evolutionary computation, genetic programming may be the most promising candidate. This is because genetic programming is built upon the modularity approach. It uses alphabets as its basic units (primitives) and uses contextfree grammar to generate all syntactically valid expressions. The grammar allows us to form primitive sentiments using alphabets, and to assemble primitive sentiments, in a hierarchical manner, in to more complex ones. Therefore, it is far more complex than just resentment or love, and is characterized by different degrees of complexity. Failure to see this complex structure may lead us to ignore the 'attitude-behavioral intention' gap, well observed in the literature on sentiment analysis (Nurse Rainbolt, Onozaka and McFadden, 2012; Lukoianova and Rubin, 2014).

The above genetic-programming route to the evolution of the system of symbols can be implemented and studied in an agent-based model. In fact, research on agent-based modeling of language evolution has already begun (Vogt and De Boer, 2010; Gong and Shuai, 2013; Lekvam, Gambäck and Bungum, 2014). ${ }^{15}$ Evolutionary computation has been also applied in some of these studies (Lekvam, 2014). We expect that in the future this line of research can be fruitfully integrated with sentiment analysis.

\section{Conflict of Interest}

On behalf of all authors, the corresponding author states that there is no conflict of interest.

\footnotetext{
15 Vogt and De Boer Vogt and De Boer (2010) further distinguish two types of agent-based models of language evolution, namely, agent-based analytical model and agent-based cognitive model. For a list of examples on each, the interested reader is referred to Vogt and De Boer (2010), p.8.
} 


\section{References}

Aggarwal C, Zhai C (2012a) An introduction to text mining. In: Aggarwal C, Zhai $\mathrm{C}$ (eds.), Mining Text Data, Chapter 1. Springer.

Aggarwal C, Zhai C (2012b) A survey of text clustering algorithms. In: Aggarwal C, Zhai C (eds.), Mining Text Data, Chapter 4. Springer.

Aghion P, Holden R (2011) Incomplete contracts and the theory of the firm: What have we learned over the past 25 years? The Journal of Economic Perspectives 25(2): 181-197.

Akhtar J, Koshul B, Awais M (2013) A framework for evolutionary algorithms based on Charles Sanders Peirce's evolutionary semiotics. Information Sciences 236: 93-108.

Al-Najjar N, Anderlini L, Felli L (2006) Undescribable events. Review of Economic Studies 73:849-868.

Angeletos, G.-M. and La'O, J. (2013), Sentiments. Econometrica, 81: 739779.

Antenucci D, Cafarella M, Levenstein M, Ré C, Shapiro M (2014) Using Social Media to Measure Labor Market Flows. NBER Working Paper, No. 20010.

Arrow K, Bernheim B, Feldstein M, McFadden D, Poterba J, Solow R (2011) 100 years of the American Economic Review: The top 20 articles. The American Economic Review 101(1):1-8.

Aubert K (1982a) Accurate predictions and fixed point theorems. Social Science Information 21 (3):323-348.

Aubert K (1982b) Accurate predictions and fixed point theorems: A reply to Simon. Social Science Information 21 (4-5):612-622.

Aubert K (1987) Fixed-point theorems and public prediction of social behavior. Advances in Applied Mathematics 8 (1):17.

Boettke P (ed.) (2000) Socialism and the Market: The Socialist Calculation Debate Revisited. 9 volumes. Routledge.

Bollen J, Mao H, Zeng X (2011) Twitter mood predicts the stock market. Journal of Computational Science 2(1):1-8.

Bozbay I, Dietrich F, Peter H (2014) Judgment aggregation in search for the truth. Games and Economic Behavior 87:571-590.

Butler D (2013) When Google got flu wrong. Nature 494:155-156.

Chen S.-H (2015) Agent-Based Computational Ecnomics: How the Idea Originated and Where It Is Going. Routledge.

Choi H, Varian H (2012) Predicting the present with Google Trends. Economic Record 88:2-9.

Cockshott W, Cottrell A (1997) Information and economics: A critique of Hayek. Research in Political Economy 18(1):177-202.

Crawford K, Gray M, Miltner K (2014) Critiquing big data: Politics, ethics, epistemology - Introduction to Special Section. International Journal of Communication 8:1663-1672.

Dietrich F (2006) Judgment aggregation: (Im)possibility Theorems. Journal of Economic Theory 126 (1):286-298.

Dredze M (2012) How social media will change public health. IEEE Intelligent Systems, 27(4):pp.81-84.

Gayo-Avello D (2012) No, you cannot predict elections with Twitter. IEEE Internet Computing $16(6): 91-94$. 
Gong T, Shuai L (2013) Computer simulation as a scientific approach in evolutionary linguistics. Language Sciences 40:12-23.

Granovetter M (1973) The strength of weak ties. American Journal of Sociology, 78(6):1360-1380.

Hart O, Moore J (1999) Foundations of incomplete contracts. Review of Economic Studies 66 (1):115-138.

Hayek F (1937) Economics and knowledge. Economica 4: 33-54.

Hayek F (1945) The uses of knowledge in society. American Economic Review 35(4): 519-530.

Hayek F (1952) The Sensory Order: An Inquiry into the Foundations of Theoretical Psychology. University of Chicago Press.

Hodgson R, Maloney J (2013) Bandwagon effects in British elections, 1885-1910. Public Choice 157:73-90.

Holland J, Holyoak K, Nisbett R (1986) Induction: Processes of Inference, Learning and Discovery (Computational Models of Cognition and Perception), MIT Press, Cambridge.

Hu X, Liu H (2012) Text anlaytics in social media. In: Aggarwal C, Zhai C (eds), Mining Text Data, Chapter 12. Springer

Jacoby J, Speller D, Kohn C (1974) Brand choice behavior as a function of information load. Journal of Marketing Research 1974 11(1): 63-69.

Jacoby J, Speller D, Berning C (1974) Brand choice behavior as a function of information load: Replication and extension. Journal of Consumer Research 1974 1: $33-42$.

Kim S, Kim D (2014) Investor sentiment from internet message postings and the predictability of stock returns. Journal of Economic Behavior and Organization 107:708-729.

Kiss Á, Simonovits G (2014) Identifying the bandwagon dffect in two-round elections. Public Choice 160:327-344.

Lavoie D (1985) Rivalry and Central Planning: The Socialist Calculation Debate Reconsidered. Cambridge: Cambridge University Press.

Lekvam T (2014) Co-evolving Language and Social Structure Using a Genetic Algorithm. Master Thesis, Department of Computer and Information Science, Norwegian University of Science and Technology.

Lekvam T, Gambäck B, Bungum L (2014) Agent-based modeling of language evolution. In Proceedings of the 5th Workshop on Cognitive Aspects of Computational Language Learning, pp. 49-54.

Li X, Xie H, Chen L, Wang J, Deng X (2014) News impact on stock price return via sentiment analysis. Knowledge-Based Systems 69:14-23.

List C (2012) The theory of judgment aggregation: An introductory review. Synthese 187 (1):179-207.

Liu B, Zhang L (2012) A survey of opinion mining and sentiment analysis. In: Aggarwal C, Zhai C (eds), Mining Text Data, Chapter 13. Springer.

Lukoianova T, Rubin V (2014) Veracity roadmap: Is big data objective, truthful and credible? Advances In Classification Research Online 24(1):4-15.

Metaxas P, Mustafaraj E, Gayo-Avello D (2011) How (not) to predict elections. Proceedings of Privacy, Security, Risk and Trust (PASSAT) and 2011 IEEE Third International Conference on Social Computing (SocialCom), 165-171.

Mian A, Sufi A, Khoshkhou N (2015) Government Economic Policy, Sentiments, and Consumption, NBER Working Paper No. 21316. 
Milgram S (1967) The small world problem. Psychology Today 2:60-67.

Mo S. Y. K., Liu A, Yang S. Y. (2015) News sentiment to market impact and its feedback effect. Available at SSRN 2567433.

Murthy D (2015) Twitter and elections: Are tweets, predictive, reactive, or a form of buzz? Information, Communication \& Society 18(7):816-831.

Netzer O, Feldman R, Goldenberg J, Fresko M (2012) Mine your own business: Market-structure surveillance through text mining. Marketing Science 31(3): 521-543.

Nenkova A, McKeown K (2012) A survey of text summarization techniques. In: Aggarwal C, Zhai C (eds), Mining Text Data, Chapter 3. Springer.

Nurse Rainbolt G, Onozaka Y, McFadden D (2012) Consumer motivations and buying behavior: The case of the local food system movement. Journal of Food Products Marketing 18(5):385-396.

O'Reilly T, Battelle J (2009) Web Squared: Web 2.0 Five Years On. O'Reilly Media.

Otnes P (2011) Simon's finals: Can self-defeating predictions be righted beforehand? Sosiologisk Årbok (1-2):109-126

Park D, Lee J (2009) eWOM overload and its effect on consumer behavioral intention depending on consumer involvement. Electronic Commerce Research and Applications 7(4): 386-398.

Polanyi M (1966) The Tacit Dimension. University of Chicago Press.

Posner R (2004) Introduction to the Becker-Posner blog. http://www.becker-posner-blog.com/archives/2004/12/introduction_to_1.html, December.

Schroder H, Driver M, Streufert S (1967) Human Information Processing. Holt, Rinehart and Winston.

Simon H (1954) Bandwagon and underdog effects and the possibility of election predictions. The Public Opinion Quarterly 18 (3):245-253.

Simon H (1971) Designing organizations for an information-rich world. In Greenberger M (ed.), Computers, Communications and the Public Interest. Johns Hopkins University Press, pp. 37-72.

Simon H (1982a) Accurate predictions and fixed point theorems: Comments. Social Science Information 21 (4-5):605-612.

Simon H (1982b) A final comment. Social Science Information 21:622-626.

Smith V (1982) Markets as economizers of information: Experimental examination of the "Hayek Hypothesis". Economic Inquiry 20(2): 165-179.

Sun Y, Deng H, Han, J (2012) Probabilistic models for text mining. In: Aggarwal C, Zhai C (eds.), Mining Text Data, Chapter 8. Springer.

Sunstein C (2008) Neither Hayek nor Habermas. Public Choice 134: 87-95.

Tetlock P (2007) Giving content to investor sentiment: The role of media in the stock market. Journal of Finance 62: 1139-1168.

Tetlock P, Saar-Tsechansky M, Macskassy S (2008) More than words: Quantifying language to measure firms' fundamentals. Journal of Finance 63: 1437-1467.

Tirole J (1999) Incomplete contracts: Where do we stand?. Econometrica 67: 741781.

Tumasjan A, Sprenger T, Sandner P, Welpe I (2010) Predicting elections with Twitter: What 140 Characters reveal about political sentiment. Proceedings of the Fourth International AAAI Conference on Weblogs and Social Media, 178185. 
Velupillai K (2010) Computable Foundations for Economics. Routledge.

Vogt P, De Boer B (2010) Language evolution: Computer models for empirical data. Adaptive Behavior 18(1):5-11.

Von Mises L (1935): Economic calculation in the socialist commonwealth. In Hayek F (ed.), Collectivist Economic Planning. Routledge and Kegan Paul.

Vriend N (2002) Was Hayek an ACE? Southern Economic Journal 68(4): 811-840. Zipf G (1936). The Psychobiology of Language. London: Routledge.

Zipf G (1949). Human Behavior and the Principle of Least Effort. New York: Addison-Wesley 